\title{
Biomarkers for the targeted therapies of non-small cell lung cancer
}

\author{
This article was published in the following Dove Press journal: \\ Current Biomarker Findings \\ 12 April 2012 \\ Number of times this article has been viewed
}

\author{
Antonio Rossi' \\ Domenico Galetta ${ }^{2}$ \\ 'Division of Medical Oncology, \\ S.G. Moscati Hospital, Avellino, Italy; \\ ${ }^{2}$ Department of Medical Oncology, \\ Giovanni Paolo II Oncology Institute, \\ Bari, Italy
}

\begin{abstract}
Targeted therapy includes new biologic agents specifically designed to selectively target molecular pathways responsible for, or that substantially drive, the malignant phenotype of cancer cells. A lot of new biologic agents have been introduced in clinical development for the treatment of advanced non-small cell lung cancer (NSCLC), but unfortunately negative results were more frequent than successes. Two pathways have been deeply studied and have led to the development of corresponding biomarkers for defining the most appropriate therapeutic approach in advanced NSCLC patients. The epidermal growth factor receptor (EGFR) pathway is targeted by tyrosine kinase inhibitors, gefitinib and erlotinib, and monoclonal antibody, cetuximab. EGFR mutation status, EGFR gene copy number determined by fluorescent in situ hybridization, and $E G F R$ protein expression determined by immunohistochemistry have been evaluated as potential markers for clinical decision making regarding anti-EGFR therapy. Among these, EGFR mutation status resulted in the most important predictive/prognostic factor for $E G F R$-tyrosine kinase inhibitor therapy. EGFR protein expression seems to be important for cetuximab plus chemotherapy treatment, but further data are needed to define its role in this setting. In the last few years, the anaplastic lymphoma kinase $(A L K)$ gene fusion is becoming an important biomarker in defining the specific NSCLC subtype to target with the corresponding inhibitor, crizotinib. To date, considering the EGFR activating mutations and the $A L K$ gene fusion, generally mutually exclusive, and the availability of the correspondent inhibitors, $20 \%$ to $50 \%$ of advanced NSCLC could now be treated in Western and Eastern countries, respectively, with a targeted therapy.
\end{abstract}

Keywords: biomarkers, EGFR, ALK, NSCLC, TKI, crizotinib

\section{Introduction}

Targeted therapy, the key phrase in cancer treatment since the start of the new millennium, refers to using agents specifically designed to selectively target molecular pathways responsible for, or that substantially drive, the malignant phenotype of cancer cells. Targeted agents are designed to be selective in their effects by modulating the activity of proteins essential for the uncontrolled growth, angiogenesis, invasiveness, and metastatic processes of malignant tumors, implying that they should cause fewer side effects on normal cells. On the contrary, most chemotherapeutic agents are relatively nonselective in their activity, although their mechanisms of action work by damaging cells undergoing mitosis, which is usually more common in malignant tumors than in most normal tissues. This has led to an increase in toxicity due to the damage of normal cells too. ${ }^{1}$
Division of Medical Oncology

S.G. Moscati Hospital, Contrada

Amoretta 8, Avellino 83100, Italy

Tel +39825203573

Fax +39825203556

Email arossi_it@yahoo.it 
Non-small cell lung cancer (NSCLC) accounts for about $85 \%$ of all new lung cancer diagnosis and includes three main histological types: adenocarcinoma, squamous cell carcinoma, and large cell carcinoma. ${ }^{2}$ At diagnosis more than $50 \%$ of patients have advanced disease for which systemic therapy is the standard of care to be used only in this stage of disease in which the targeted therapy has been investigated, also due to a plateau of effectiveness reached by standard chemotherapy. A lot of new biologic agents have been introduced in clinical investigation for the treatment of advanced NSCLC, but unfortunately negative results have been more frequent than successes. In fact, despite these new biologic agents being developed to block a specific target, when investigated in clinical trials no selection of patients was requested. This has led to a misunderstanding of the real therapeutic power of these drugs. To select patients in order to optimize the effect of targeted therapy, predictive and/or prognostic biomarkers of activity should be identified. The main goal of targeted therapy ought to be the so-called "personalized medicine," meaning the possibility to treat NSCLC with specific biologic characteristics representing the target for a specific inhibitor drug.

The current review will provide an update on the predictive role of the two most clinically relevant molecular biomarkers in NSCLC to date, ie, epidermal growth factor receptor $(E G F R)$ and anaplastic lymphoma kinase $(A L K)$ gene translocations.

\section{EGFR}

$E G F R$, also known as ErbB-1/HER1, is the first of four members of the ErbB family of cell membrane receptors which are important mediators in cell growth, differentiation, and survival. ${ }^{3}$ The EGFR binds with a high affinity to several ligands, such as EGF, amphiregulin, and transforming growth factor- $\alpha$, and it is highly expressed (about $40 \%-80 \%$ ) in NSCLC. EGFR is the target for two classes of inhibitors: (1) the small-molecule $E G F R$-tyrosine kinase inhibitors (TKIs), such as gefitinib and erlotinib, administered orally on a daily basis, which inhibit the $E G F R$ activity by competing with adenosine triphosphate for the adenosine triphosphatebinding site localized on the EGFR intracellular domain; and (2) the monoclonal antibodies, such as cetuximab, administered intravenously on a weekly basis, directed against the extracellular domain of the EGFR, blocking ligand binding and receptor activation. ${ }^{4}$

$E G F R$ mutation status, EGFR gene copy number determined by fluorescent in situ hybridization (FISH), and EGFR protein expression determined by immunohistochemistry
(IHC) have been evaluated as potential markers for clinical decision making regarding anti-EGFR therapy.

\section{EGFR mutation status}

The identification of its activating somatic mutations in the $E G F R$ gene provided the first sight of a clinically relevant NSCLC oncogene..$^{5-7}$ These mutations are usually found in exons 18-21 and are either point mutations or in-frame small deletions or insertions. The most common mutations include an in-frame deletion of exon 19 (about $45 \%-50 \%$ of mutations) and the L858R point mutation in exon 21 (about $40 \%-45 \%$ of mutations) ${ }^{8-10}$ EGFR mutations are more common in patients with adenocarcinoma histology, women, Asians, and never smokers, and are detected in approximately $10 \%-15 \%$ of all NSCLCs in Caucasians and $20 \%-30 \%$ of all NSCLCs in East Asians, with prevalence increasing to $50 \%$ or more in never smokers. ${ }^{8}{ }^{89}$ The presence of activating mutations trigger the $E G F R$-signaling pathway in the absence of ligands, and promote EGFR-mediated prosurvival and antiapoptotic signals through downstream targets such as phosphatidylinositol-3-kinases/Akt, extracellular signalregulated kinase/mitogen-activated protein kinase, and signal transducer and activator of transcription. ${ }^{11,12}$ However, EGFR mutations also alter the tyrosine kinase pocket of the receptor to a degree that enhances the sensitivity to adenosine triphosphate-competitive EGFR inhibitors. ${ }^{13}$ This is why patients affected by NSCLC harboring an activating EGFR mutation are particularly sensitive to EGFR-TKI therapy.

At the beginning, gefitinib and erlotinib were clinically investigated within Phase III randomized trials in unselected patients affected by advanced NSCLC in both first-line and second-line therapy, in combination with standard platinumbased chemotherapy ${ }^{14-17}$ or administered as single agent. ${ }^{18-20}$ Unfortunately, overall results were negative with the exception of the trial in which erlotinib was compared with placebo in previously treated NSCLC patients (BR.21 trial). In this trial, the primary endpoint was met with erlotinib improving overall survival (OS; 6.7 months versus 4.7 months, respectively; hazard ratio [HR] 0.70; $P<0.001)$. Erlotinib also reported a better objective response rate (ORR 8.9\% versus $>1 \%$, respectively; $P<0.001)$ and progression-free survival (PFS; 2.2 months versus 1.8 months, respectively; HR $0.61 ; P<0.001) .{ }^{19}$ These results led to the marketing of erlotinib for the treatment of unselected previously treated NSCLC patients worldwide. The relative limited activity reported by gefitinib and erlotinib in non-EGFR genotyped, or unselected, NSCLC patients was counterbalanced by the significant clinical and radiographic responses registered 
through their administration in most patients whose tumors harbor EGFR-activating mutations when given as first-line, second-line, or subsequent lines of therapy. ${ }^{21}$

Retrospective analyses from randomized trials reported that some clinical characteristics were associated with high activity of EGFR-TKIs. Thus, two Phase III randomized trials compared gefitinib versus chemotherapy as first-line treatment of advanced NSCLC patients selected for clinical characteristics. ${ }^{22-24}$ As reported above, the possibility of detecting an EGFR activating mutation is higher in the patients with these clinical characteristics.

The IPASS (Iressa ${ }^{\circledR}$ Pan-Asia Study) study is a randomized Phase III trial comparing gefitinib $250 \mathrm{mg}$ /day to carboplatin plus paclitaxel in 1217 Asian patients. Adenocarcinoma, including bronchioloalveolar carcinoma, and either never smokers or former light smokers were the criteria for patient selection. Gefitinib scored better than chemotherapy in terms of PFS, which was the primary endpoint of the trial (HR for PFS was $0.74 ; 95 \%$ confidence interval [CI]: $0.65-$ $0.85 ; P<0.0001)$. However, the median PFS was similar (5.7 months versus 5.8 months for gefitinib and chemotherapy, respectively) due to the crossing shape of the Kaplan-Meier curves, which showed a better outcome with chemotherapy in the first 6 months, but subsequently favored gefitinib. ORR was $43 \%$ for the gefitinib arm versus $32.2 \%$ for chemotherapy (odds ratio [OR] 1.59; 95\% CI: 1.25-2.01; $P=0.001$ ), although no differences were reported in OS (HR for death in the gefitinib group was $0.90 ; 95 \% \mathrm{CI}: 0.79-1.02 ; P=0.109$ ), with a median OS of 18.8 and 17.4 months, respectively. ${ }^{22,23}$ Preplanned retrospective analysis was performed according to molecular markers: EGFR mutation status, EGFR gene copy number, and $E G F R$ protein expression. A total of 437 patients were evaluable for EGFR mutation status. Gefitinib scored significantly longer in PFS among the 261 patients with $E G F R$ mutation-positive tumors than among those who received carboplatin/paclitaxel (HR for progression or death 0.48 ; 95\% CI: $0.36-0.64 ; P<0.001)$, whereas in the subgroup of 176 patients who were EGFR mutation-negative, PFS was significantly longer among those who received chemotherapy (HR for progression or death with gefitinib 2.85; 95\% CI: 2.05-3.98; $P<0.001)$. When analyzing each activating mutation, a slight difference was reported in the outcomes related to the exon 19 deletion (HR with gefitinib 0.38; 95\% CI: 0.26-0.56) and L858R mutation (HR with gefitinib 0.55 ; 95\% CI: $0.35-0.87$ ) subgroups. ORR in EGFR mutation-positive patients was $71.2 \%$ for gefitinib versus $47.3 \%$ for carboplatin/paclitaxel (OR 2.75; 95\% CI: $1.65-4.60 ; P<0.001)$, and $1.1 \%$ versus $23.5 \%$ in $E G F R$ mutation-negative patients, respectively (OR 0.04; 95\% CI: $0.01-0.27 ; P=0.0013)$. Surprisingly, a greater ORR was reported for patients harboring the exon 19 mutation (ORR $84.8 \%$ for gefitinib and $43.2 \%$ for chemotherapy; OR 7.23; 95\% CI: 3.19-16.37) and not the L858R mutation (ORR $60.9 \%$ and $53.2 \%$, respectively; OR 1.41 ; $95 \%$ CI: $0.65-3.05)$. No difference in survival in EGFR mutation-positive patients (median OS of 21.6 months for gefitinib versus 21.9 months for chemotherapy, respectively; HR 1.00; 95\% CI: 0.76-1.33; $P=0.99$ ) or $E G F R$ mutation-negative subgroup (median OS of 11.2 months for gefitinib versus 12.7 months for chemotherapy; HR 1.18; 95\% CI: $0.86-1.63$; $P=0.309$ ) was reported. The absence of a survival advantage for the gefitinib arm could be explained by the administration of a second-line therapy with about $50 \%$ of patients who received the crossover treatment. ${ }^{22,23}$

In the second randomized Phase III trial (First-SIGNAL study), gefitinib was compared to cisplatin/gemcitabine in 309 patients clinically selected with eligibility criteria similar to those used in the IPASS trial. ${ }^{24} \mathrm{OS}$, the primary endpoint of the study, was similar in both groups, with 22.3 months for gefitinib versus 22.9 months for chemotherapy (HR 0.932; 95\% CI: 0.716-1.213; $P=0.604$ ). About $30 \%$ of enrolled patients were analyzed for EGFR mutation status with an overall EGFR mutation rate of $43.8 \%$ (42 out of 96 patients). OS for the gefitinib arm was 27.2 months in the mutation-positive group $(\mathrm{n}=26)$ and 18.4 months in the mutation-negative group $(n=27)$, while it was similar for the chemotherapy arm with 25.6 months in the mutation-positive group and 21.9 months in the mutationnegative group. However, there was also a high proportion of crossover at disease progression in the chemotherapy arm, with $75 \%$ of patients receiving a second-line EGFR-TKI PFS was 5.8 months in the gefitinib arm versus 6.4 months for the chemotherapy arm (HR 1.198; 95\% CI: 0.944-1.520; $P=0.138$ ), while the curve crossed over around the median time. In the gefitinib arm, PFS was significantly shorter in the mutation-negative subgroup than in the mutationpositive subgroup with a median time of 2.1 months versus 8.0 months, while there was no difference in the cisplatin/ gemcitabine arm (6.3 months versus 6.4 months). ${ }^{24}$ ORR was $55.4 \%$ for the gefitinib arm and $46 \%$ for the cisplatin/ gemcitabine group (OR 1.455; 95\% CI: 0.929-2.278; $P=0.101)$. In $E G F R$ mutation-positive patients, ORR was $84.6 \%$ for gefitinib versus $37.5 \%$ for chemotherapy (OR 9.167; 95\% CI: $2.109-39.847 ; P=0.002$ ), and $25.9 \%$ for gefitinib versus $51.9 \%$ for chemotherapy in EGFR mutation-negative patients (OR 0.325; 95\% CI: 0.103-1.021; $P=0.051$ ). 
Of interest is the different ORRs obtained with gefitinib in EGFR mutation-negative patients between the FirstSIGNAL and the IPASS trial (25.9\% and $1.1 \%$, respectively). A possible explanation could be that mutation tests were not centralized in the First-SIGNAL study, and this could have determined a higher false negative rate.

Four randomized Phase III trials compared gefitinib ${ }^{25-27}$ or erlotinib ${ }^{28,29}$ with chemotherapy as first-line treatment of NSCLC harboring EGFR-activating somatic mutations. In the WJTOG (West Japan Thoracic Oncology Group) trial, 172 EGFR mutation-positive patients were randomized to receive gefitinib or cisplatin plus docetaxel. The median PFS, the primary endpoint, was reached with 9.2 months for the gefitinib arm and 6.3 months the chemotherapy group (HR 0.489 ; 95\% CI: $0.336-0.710 ; P<0.0001)$. ORR was significantly higher in the gefitinib arm when compared with chemotherapy (62.1\% versus $32.2 \%$, respectively). OS was 30.9 months in the experimental arm, and still not reached in the control arm. ${ }^{25}$ In the NEJ002 (North East Japan 002) study, 228 patients were randomized to receive gefitinib or carboplatin/paclitaxel, showing a superiority for gefitinib in terms of PFS, the primary endpoint, with a median time of 10.8 months versus 5.4 months, respectively (HR 0.30; 95\% CI: $0.22-0.41 ; P<0.001$ ). ORR was $73.7 \%$ and $30.7 \%$, respectively $(P<0.001)$. No difference was reported in terms of survival, with a median time of 27.7 months in the gefitinib arm and 26.6 months in the chemotherapy group $(P=0.48) .^{26,27}$

In the OPTIMAL trial, erlotinib (150 mg daily) was compared with carboplatin/gemcitabine in 154 Chinese patients with advanced NSCLC harboring EGFR mutations. The primary endpoint was PFS, which was 13.1 months with erlotinib and 4.6 months with chemotherapy (HR 0.16; 95\% CI: $0.10-0.26 ; P<0.0001)$. ORR was $83 \%$ with erlotinib and $36 \%$ with chemotherapy $(P<0.0001)$. The only study ever performed in European countries and investigating an EGFR-TKI in patients with advanced NSCLC harboring an activating EGFR mutation is the EURTAC (European Erlotinib Versus Chemotherapy) trial. In this study, erlotinib was compared with a platinum-based doublet in 174 patients. The main endpoint in this trial was also PFS, which was significantly superior in the erlotinib arm (9.7 months) compared with the chemotherapy group (5.2 months; HR 0.37 ; 95\% CI: $0.25-0.54$ ). ORR was $58 \%$ and $15 \%$, respectively. ${ }^{29}$ In both the OPTIMAL and EURTAC trials, the survival data were not mature. ${ }^{28,29}$

To date, according to the above mentioned results, the European Medicine Agency granted marketing authorization for gefitinib for the treatment of locally advanced or metastatic NSCLC with sensitizing mutations of the EGFR gene across all lines of therapy, while erlotinib has not been granted in this setting yet.

In all of these trials, the safety profile, the control of disease-related symptoms, and the global quality of life was better in the group of patients treated with EGFR-TKIs. Skin rash and diarrhea are the most frequent toxicities related to the EGFR-TKI therapy, while less common are nausea, vomiting, anorexia, and transaminase elevations, which usually are mild-moderate and regress after discontinuation of therapy. Among potentially life-threatening events, interstitial lung disease has been reported. However, it was relatively uncommon in patients treated with EGFR-TKIs, as reported by the randomized Phase III trials mentioned above.

Overall, the presence of an EGFR mutation resulted in a strong predictor of a better outcome with EGFR-TKIs. Despite this consideration, no survival improvement was reported. A valid explanation is that the percentage of crossover was very high, irremediably influencing the survival results; nevertheless, in all reported studies the median OS for both arms ranged between 18-30 months, results which were never observed in trials addressing advanced NSCLC patients.

The role of EGFR mutation status has also been investigated for cetuximab therapy. A randomized Phase III trial, BMS099, compared carboplatin/taxanes (paclitaxel or docetaxel) with or without cetuximab in 676 chemotherapynaïve patients with advanced NSCLC, without restrictions based on histology or EGFR expression. The primary endpoint was median PFS, assessed by independent radiologic review committee, which was 4.4 months for cetuximab plus chemotherapy and 4.24 months for chemotherapy alone (HR 0.902; 95\% CI: 0.761-1.069; $P=0.236$ ). Median OS was 9.69 months versus 8.38 months, respectively (HR 0.890; 95\% CI: 0.754-1.051; $P=0.169$ ). ORR by independent radiologic review committee was $25.7 \%$ for cetuximab plus chemotherapy versus $17.2 \%$ for chemotherapy alone $(P=0.007) .{ }^{30}$ Retrospective analyses evaluated the role of EGFR mutations. A total of 166 patients were evaluable for the $E G F R$ mutational status and 17 (10.2\%) resulted positive. Cetuximab plus chemotherapy did not significantly affect any outcome. PFS in patients with EGFR wild-type was 5.1 months in the cetuximab-based arm versus 4.6 months in the chemotherapy alone group (HR 0.95; 95\% CI: 0.66-1.35; $P=0.76$ ). In patients $E G F R$ mutation-positive, PFS was 6.1 months versus 6.4 months, respectively (HR 1.17; 95\% CI: 0.36-3.77; $P=0.79$ ). Median OS in patients with EGFR wild-type was the same 
in both groups (9.8 months; HR 0.91; 95\% CI: 0.64-1.29; $P=0.61$ ), while in $E G F R$ mutation-positive patients it was 17.6 months in the cetuximab-based arm versus 20 months in the chemotherapy alone group (HR $1.62 ; 95 \% \mathrm{CI}$ : $0.54-4.88 ; P=0.38)$. ORR tended to be higher in EGFR mutation-positive patients receiving cetuximab plus chemotherapy ( $50 \%$ versus $11.1 \% ; P=0.13)$; a similar pattern was found in the $E G F R$ wild-type group (32.4\% versus $21.8 \%$ ). ${ }^{31}$ A randomized Phase III study (FLEX trial) enrolled 1125 patients affected by EGFR-expressing NSCLC to receive, as first-line therapy, cisplatin/vinorelbine with or without cetuximab. OS was the primary endpoint, which was reached with cetuximab, improving median OS significantly compared with chemotherapy alone (11.3 months versus 10.1 months, respectively; HR 0.871 ; $95 \%$ CI: $0.762-0.996$; $P=0.044)$. The median PFS was 4.8 months in both groups (HR 0.943 ; 95\% CI: $0.825-1.077 ; P=0.39$ ). ORR was $36 \%$ in the cetuximab plus chemotherapy group and $29 \%$ in the chemotherapy arm. The main cetuximab-related toxicity was acne-like rash which was grade 3 in $10 \%$ of cases. ${ }^{32}$ Also for this trial, retrospective analyses were performed according to several biomarkers. EGFR kinase domain mutation status was assessable in 436 (39\% of all cases) patients. Activating EGFR mutations were identified as indicators of good prognosis in both groups of treatment. In fact, $E G F R$ mutation-positive and $E G F R$ mutation-negative patients treated with chemotherapy plus cetuximab reported a median OS of 17.5 months and 8.5 months, respectively (HR 0.52; 95\% CI: $0.32-0.84 ; P=0.0063$ ), while median OS in the chemotherapy group was 23.8 months and 10 months, respectively (HR 0.35 ; 95\% CI: $0.21-0.59, P<0.0001$ ). PFS in the EGFR mutation-positive and the EGFR mutation-negative patients treated with chemotherapy plus cetuximab was 5.4 months and 4.2 months (HR 0.78; 95\% CI: $0.51-1.18 ; P=0.24)$, while in the chemotherapy arm it was 5.6 months and 4.9 months, respectively (HR 0.92; 95\% CI: $0.61-1.38 ; P=0.68)$. ORR in the $E G F R$ mutationpositive and the $E G F R$ mutation-negative patients treated with chemotherapy plus cetuximab was $46.4 \%$ and $31.7 \%$ (OR 1.87; 95\% CI: $0.83-4.17 ; P=0.13$ ), while in the chemotherapy arm it was $38.9 \%$ and $28.5 \%$, respectively (OR 1.60; 95\% CI: $0.76-3.35 ; P=0.21$ ). ${ }^{33}$

Looking at these results, contrasting with what was reported for EGFR-TKI therapy, EGFR mutation status was not predictive for the efficacy of chemotherapy plus cetuximab, but it was predictive of an overall better prognosis. This finding is consistent with the role of being a prognostic factor in NSCLC.

\section{EGFR gene copy number}

Several retrospective analyses evaluated the clinical results of $E G F R$ inhibitors using the predictive markers $E G F R$ gene copy number detected by FISH. A retrospective analysis demonstrated that EGFR FISH-positive patients when treated with gefitinib had a significantly longer time to progression and survival than patients EGFR FISH-negative. ${ }^{34}$ For the trial comparing carboplatin/paclitaxel with or without erlotinib (TRIBUTE trial), the results of EGFR FISH were available for 245 of the 1059 patients who participated in the study. In FISH-positive patients, time to progression was significantly longer (HR 0.59; 95\% CI: $0.35-0.99 ; P=0.043$ ) for patients on erlotinib, but there was no difference in OS, nor was there any difference in time to progression or OS for the FISHnegative patients. ${ }^{35}$ In the BR.21 trial, EGFR FISH-positive patients treated with erlotinib reported an ORR significantly higher $(P=0.02)$ when compared with FISH-negative patients ( $21.4 \%$ versus $4.8 \%$, respectively). The survival benefit for erlotinib compared with placebo was significant in FISHpositive patients (HR 0.43 ; $95 \%$ CI: $0.23-0.78 ; P=0.004$ ), but not in FISH-negative patients (HR 0.80; 95\% CI: 0.49-1.29; $P=0.35$ ). ${ }^{36}$ In the ISEL (Iressa Survival Evaluation in Lung Cancer) trial comparing gefitinib versus placebo in unselected previously treated NSCLC patients, a total of 114 patients (30.8\%) were EGFR FISH-positive and achieved a significantly better OS with gefitinib compared with placebo than $E G F R$ FISH-negative patients $(P=0.045)$. Median OS among EGFR FISH-positive patients was 8.3 months for gefitinib and 4.5 months for placebo. No apparent difference in OS between gefitinib and placebo was observed in EGFR FISHnegative patients (HR 1.16; 95\% CI: 0.81-1.64; $P=0.417$ ). EGFR FISH-positive patients achieved better ORR and time to failure than EGFR FISH-negative patients. ${ }^{37}$ However, in both studies, EGFR FISH status was not an independent predictive factor in the multivariate analysis. This may in part be due to the lack of sufficient tumor samples for the majority of patients in these trials. In the INTEREST (Iressa NSCLC Trial Evaluating Response and Survival against Taxotere ${ }^{\circledR}$ ) trial comparing gefitinib and docetaxel in unselected previously treated NSCLC patients, 374 out of 1466 randomized patients were assessable for $E G F R$ FISH status. A total of 174 (47\%) assessable patients were EGFR FISH-positive. OS was superior for gefitinib versus docetaxel in $E G F R$ FISHpositive patients (HR 1.09; $95 \% \mathrm{CI}: 0.78-1.51 ; P=0.62$ ). OS outcomes in EGFR FISH-negative patients were also similar for both treatments (HR 0.93; 95\% CI: $0.68-1.26 ; P=0.64$ ). Gefitinib was similar to docetaxel in terms of PFS in EGFR FISH-positive (HR 0.84; 95\% CI: 0.59-1.19; $P=0.33$ ) and 
EGFR FISH-negative patients (HR 1.30; 95\% CI: 0.93-1.83; $P=0.12)$. ORR was higher in EGFR FISH-positive patients receiving gefitinib compared with those receiving docetaxel (13.0\% versus $7.4 \% ; P=0.04) .{ }^{38}$

A prospective Phase II study (ONCOBELL trial), selected patients who were EGFR FISH-positive and were phosphoAkt positive or never smokers. Of the 183 patients who were evaluated, 42 patients were enrolled in the trial and treated with gefitinib. ORR observed was $47.6 \%$ (68\% in EGFR FISH-positive), whereas no responses were observed in never smokers who were also negative for EGFR FISH and mutation. The median time to progression was 6.4 months and the 1-year survival rate was $64.3 \%$. EGFR mutations were detected in 24 patients $(66.8 \%)$ and ORR observed in those patients was $62.5 \% .{ }^{39} \mathrm{~A}$ randomized Phase II trial performed in elderly patients ( $\geq 70$ years) compared gefitinib and vinorelbine as first-line therapy. The exploratory endpoint included the association of EGFR gene copy number with gefitinib and vinorelbine activity. Of the 196 patients who underwent random assignment, 191 patients provided a tumor sample and, of these, 158 were assessable for $E G F R$ gene copy number detection. Surprisingly, in the 54 EGFR FISHpositive patients, those treated with vinorelbine achieved better PFS and survival outcomes than patients treated with gefitinib: HR was 3.13 for gefitinib (95\% CI: 1.45-6.76) versus 2.88 for vinorelbine (95\% CI: $1.21-6.83)$. Furthermore, patients who were FISH-positive and who were treated with gefitinib had a nonsignificant trend toward poorer PFS and OS outcomes compared with patients who were FISH-negative and who were treated with gefitinib: HR for FISH-positive versus FISH-negative patients who were treated with gefitinib was 1.31 (95\% CI: 0.77-2.22) for PFS and 1.61 (95\% CI: 0.87-3.01) for OS. On the other hand, patients who were FISH-positive and who were treated with vinorelbine had a nonsignificant trend toward improved PFS and OS outcomes than patients who were FISH-negative and who were treated with vinorelbine: HR for FISH-positive versus FISH-negative patients who were treated with vinorelbine was 0.77 (95\% CI: $0.43-1.39$ ) for PFS and 0.52 (95\% CI: $0.25-1.10)$ for OS. ${ }^{40}$

In the IPASS trial, the EGFR gene copy number was evaluable in 406 patients. In patients EGFR FISH-positive $(\mathrm{n}=249)$, PFS was significantly longer with gefitinib versus carboplatin/ paclitaxel (HR 0.66; 95\% CI: $0.50-0.88 ; P=0.005$ ). ORR favored gefitinib (58.9\% versus $44.8 \%$; OR 1.79 ; 95\% CI: 1.08-2.96; $P=0.024)$. In patients $E G F R$ FISH-negative ( $\mathrm{n}=157$ ), PFS was longer (HR 1.24; 95\% CI: 0.87-1.76; $P=0.237)$ and ORR was higher $(26.3 \%$ versus $22.2 \%$; OR 0.80 ; 95\% CI: $0.38-1.68 ; P=0.558)$ with carboplatin/paclitaxel versus gefitinib. However, 190 (78\%) EGFR FISH-positive patients also harbored EGFR mutations. Of the $153 E G F R$ FISH-negative patients, only 51 (33\%) were also EGFR mutation-positive. PFS was significantly shorter with gefitinib versus carboplatin/paclitaxel in EGFR FISH-positive patients in the absence of a coexisting EGFR mutation ( $\mathrm{n}=55$; HR 3.85; 95\% CI: 2.09-7.09), although patients with EGFR mutation achieved significantly longer PFS with gefitinib versus carboplatin plus paclitaxel, irrespective of whether they were EGFR FISH-positive (HR 0.48; 95\% CI: $0.34-0.67 ; \mathrm{n}=190$ ) or EGFR FISH-negative (HR 0.51; 95\% CI: $0.25-1.04 ; n=51)$. No survival difference for gefitinib versus chemotherapy in EGFR FISH-positive (HR 1.03; 95\% CI: $0.78-1.37 ; P=0.816)$ or $E G F R$ FISH-negative patients (HR 1.30; 95\% CI: 0.92-1.85; $P=0.137$ ) was reported. ${ }^{23}$

A systematic review and meta-analysis, including 22 trials, assessed the EGFR gene copy number as a potential marker of OS for patients affected by NSCLC and treated with EGFR-TKIs. The EGFR FISH-positive status was associated with increased OS (HR 0.77; 95\% CI: 0.66-0.89; $P=0.001$ ), PFS (HR 0.60; 95\% CI: $0.46-0.79 ; P<0.001$ ), and time to progression (HR $0.50 ; 95 \% \mathrm{CI}: 0.28-0.91 ; P=0.02$ ). Of interest was that among predominantly white populations EGFR FISH-positive status was strongly associated with improved OS (HR 0.70; 95\% CI: 0.59-0.82; $P<0.001$ ), whereas it did not influence OS in East Asians (HR 1.11; 95\% CI: $0.82-1.50 ; P=0.50)$. This difference was statistically significant $(P=0.02) .{ }^{41}$

Overall, these contrasting results suggest that the predictive value of $E G F R$ gene copy number for outcomes benefit with gefitinib was probably driven by the overlap of the concurrent high incidence of EGFR mutation-positive status in this subgroup.

Concerning the possible relationship between cetuximab and EGFR copy number, retrospective analysis from the two main randomized Phase III trials (BMS009 and FLEX) reported the following results. In the BMS099 trial, 54 of 104 evaluable patients (51.9\%) were EGFR FISH-positive. The addition of cetuximab to carboplatin/taxane did not significantly affect PFS in the FISH-positive group (5.4 months in both arms; HR 1.54; 95\% CI: $0.81-2.93 ; P=0.18$ ) or in the FISH-negative group (4.3 months versus 3.8 months; HR 0.65 ; 95\% CI: $0.35-1.18 ; P=0.15)$. In the cetuximab plus chemotherapy group, no PFS difference was evident between patients in the FISH-positive and FISH-negative groups (HR 0.99; $P=0.97$ ). Patients with FISH-positive tumors treated with chemotherapy had significantly longer PFS than those with FISH-negative tumors (HR 1.41; $P=0.007)$. 
Patients with EGFR FISH-positive tumors had significantly shorter OS with cetuximab plus chemotherapy than with chemotherapy alone (8.6 months versus 12.5 months; HR 1.92; 95\% CI: $1.05-3.54 ; P=0.03)$, whereas OS did not differ by treatment in patients with FISH-negative tumors (7.4 months in both groups; HR 0.84; 95\% CI: $0.47-1.52 ; P=0.57)$. Patients with FISH-positive tumors had longer OS than those with FISH-negative tumors when treated with chemotherapy alone (HR 0.48; $P=0.017$ ), but not when treated with cetuximab plus chemotherapy (HR $1.07 ; P=0.81$ ). In the $E G F R$ FISH evaluable population overall, ORR was higher with cetuximab plus chemotherapy than with chemotherapy alone $(34.0 \%$ versus $24.0 \%$ ), as in patients with FISH-positive (37.0\% versus $22.2 \% ; P=0.37)$ and FISH-negative $(30.8 \%$ versus $16.7 \%$; $P=0.33$ ) tumors. ${ }^{31}$ In the FLEX trial, tumor EGFR gene copy number was analyzed in 330 of 1125 (29\%) patients. In the EGFR FISH-positive patients, the addition of cetuximab to cisplatin plus vinorelbine did not improve any outcomes, with median OS of 11.6 months and 9.9 months (HR 0.85; 95\% CI: $0.56-1.29 ; P=0.44)$, median PFS of 4.2 months and 4.4 months (HR 0.80; 95\% CI: $0.51-1.25 ; P=0.33$ ), and ORR of $36.7 \%$ and $26.4 \%$, respectively, (OR 1.62; 95\% CI: $0.70-3.76 ; P=0.26$ ) for cetuximab plus chemotherapy and chemotherapy alone. No differences in any outcomes were reported in the EGFR FISH-negative patients, with a median OS of 10.6 months and 10 months (HR 0.91 ; 95\% CI: 0.65 $1.26 ; P=0.56)$, median PFS of 4.2 months and 5.2 months (HR 1.05; 95\% CI: $0.75-1.47 ; P=0.77)$, and ORR of $32.9 \%$ and $34.7 \%$, respectively (OR $0.92 ; 95 \%$ CI: $0.49-1.72 ; P=0.80$ ) for cetuximab plus chemotherapy and chemotherapy alone. ${ }^{33}$ In both these large randomized trials, increased EGFR gene copy number was not a predictive or prognostic biomarker for cetuximab activity.

Overall, when considering EGFR gene copy number as a potential marker of EGFR inhibitor therapy, it is important to take into account that its assessment in clinical trials varies greatly. This may be due to different patient populations, different study designs, and variability in the methods used by different laboratories for reading and interpreting FISH. Thus, adequate assay reproducibility is mandatory, and therefore guidelines have been produced to provide information on the inclusion/exclusion criteria, reading and scoring of slides, assessment of signal clusters, and assessment of borderline cases. ${ }^{42}$

\section{EGFR protein expression}

$E G F R$ protein expression assessed by IHC was the first biological marker to be retrospectively explored in cohorts of
NSCLC patients treated with EGFR-TKIs. In the BR.21 study, tumor samples were available for 325 out of 731 patients treated on the trial. EGFR IHC-positive patients treated with erlotinib had a significant OS improvement when compared with $E G F R$ IHC-positive patients who received placebo (HR 0.68; 95\% CI: $0.49-0.95 ; P=0.02$ ). No difference was observed among patients who were EGFR IHC-negative. However, EGFR IHC was not an independent predictor for OS in the multivariate analysis, with a negative interaction test between EGFR expression and treatment effect. ${ }^{43}$ On the other hand, in the ISEL trial, EGFR IHC-positive patients achieved significantly better OS with gefitinib versus placebo than EGFR IHC-negative patients (interaction test, $P=0.049$ ). The OS benefit for $E G F R$ IHC-positive patients treated with gefitinib was slightly higher than the overall study population (HR 0.77; 95\% CI: 0.56-1.08; $P=0.126$ ), with no evidence of an OS benefit among patients with $E G F R$ protein-negative tumors who received gefitinib (HR 1.57; 95\% CI: $0.86-2.87 ; P=0.140) .{ }^{37}$

In the IPASS trial, 365 patients were evaluable for the $E G F R$ protein expression status. PFS was significantly longer for gefitinib versus carboplatin/paclitaxel in patients with EGFR IHC-positive tumors $(\mathrm{n}=266$; HR 0.73; 95\% CI: $0.55-0.96 ; P=0.024)$, while no difference was reported in patients with EGFR IHC-negative tumors (n = 99; HR 0.97; 95\% CI: $0.64-1.48 ; P=0.893)$. ORRs were similar between the gefitinib and carboplatin/paclitaxel groups for patients with either EGFR IHC-positive (51.5\% versus $41.8 \%$; OR 1.49; 95\% CI: $0.92-2.42 ; P=0.109)$ or EGFR IHCnegative (34.0\% versus $26.1 \%$; OR 1.44 ; 95\% CI: $0.60-3.47$; $P=0.415)$ tumors. No difference in OS between gefitinib and chemotherapy in patients with EGFR IHC-positive (HR 1.05; 95\% CI: $0.80-1.37 ; P=0.731)$ or EGFR IHC-negative (HR 1.09; 95\% CI: $0.70-1.70 ; P=0.692$ ) tumors was reported. ${ }^{23}$

Although conflicting, these data are provocative for positive $E G F R$ protein expression as a predictive molecular factor. However, these data are extrapolated retrospectively and are based on relatively small numbers of patients who had available tumor tissue. Further investigations should better clarify the role of $E G F R$ protein expression as a biomarker for EGFR-TKI therapy and the relationship with the concurrent presence of $E G F R$ activating mutations.

The predictive role of EGFR protein expression seems to be clearer when administering cetuximab therapy. In the BMS099 trial, 17 (11.5\%) out of 148 patients were EGFR IHC-negative. PFS results were comparable in the EGFR IHCpositive subgroup with a median of 4.6 months for cetuximab plus carboplatin/taxane versus 4.5 months for chemotherapy alone (HR 1.15; 95\% CI: $0.78-1.68 ; P=0.48$ ) and in the 
subset with EGFR IHC-negative tumors (4.1 months versus 6.4 months, respectively; HR 1.17; 95\% CI: 0.37-3.72; $P=0.79$ ). Adding cetuximab to chemotherapy did not significantly affect $O S$ in patients with $E G F R$ IHC-positive tumors, with a median OS of 8.3 months for the cetuximab-based group and 9.7 months for chemotherapy alone arm (HR 1.02; 95\% CI: $0.71-1.48 ; P=0.91$ ) or in the small subset of patients with EGFR IHC-negative tumors (median OS, 11.2 months versus 17.6 months, respectively; HR 1.86; $95 \%$ CI: $0.57-6.11 ; P=0.30)$. No significant ORR differences between cetuximab plus chemotherapy and chemotherapy alone arms were observed either in patients with $E G F R$ IHCpositive tumors $(31.8 \%$ versus $21.5 \% ; P=0.24)$ or with $E G F R$ IHC-negative tumors (18.2\% versus $33.3 \%$, respectively; $P=0.58) .{ }^{31}$ Very interesting are the results reported by the FLEX trial, in which patients tumor EGFR expression data were used to generate an IHC score on a continuous scale of 0-300 with a defined threshold of 200. Treatment outcome was analyzed in patients with low (IHC score $<200$ ) and high (IHC score $\geq 200$ ) tumor EGFR expression. Tumor EGFR IHC data were available for 1121 of 1125 (99.6\%) patients randomized in this trial. High EGFR expression was scored for $345(31 \%)$ evaluable patients and low for $776(69 \%)$ patients. For patients in the high EGFR expression group, OS was longer in the cetuximab plus cisplatin plus vinorelbine group than in the chemotherapy alone arm, with a median OS of 12.0 months versus 9.6 months, respectively (HR 0.73 ; 95\% CI: $0.58-0.93 ; P=0.011)$. No OS benefit for patients in the low EGFR expression group was reported with a median OS of 9.8 months versus 10.3 months, respectively (HR 0.99 ; 95\% CI: $0.84-1.16 ; P=0.88)$. A treatment interaction test assessing the difference in the HR for OS between the $E G F R$ expression groups suggest a predictive value for $E G F R$ expression $(P=0.044)$. These results suggest that high $E G F R$ expression is a tumor biomarker that can predict OS benefit from the addition of cetuximab to first-line chemotherapy in patients with advanced NSCLC. ${ }^{44}$

Overall, further trials are needed to evaluate the assessment of EGFR expression to select advanced NSCLC patients who could benefit from cetuximab plus chemotherapy firstline therapy offering a personalized treatment approach in this setting.

\section{ALK}

In the last few years, a new and promising target has emerged for the treatment of advanced NSCLC patients. $A L K$ is a transmembrane receptor tyrosine kinase in the insulin receptor superfamily. About $2 \%-7 \%$ of patients with
NSCLC have tumors with an inversion in the short arm of chromosome 2 (inv [2][p21p23]) which results in the fusion of exons 1-13 of the echinoderm microtubule-associated protein-like 4 (EML4) gene with exons 20-29 of the $A L K$ gene, leading to the production of an $E M L 4-A L K$ fusion tyrosine kinase which is involved in cell proliferation, differentiation, and antiapoptosis. ${ }^{45} E M L 4-A L K$ also contains the hydrophobic protein domain of $E M L 4$, which is critical for dimerization of $E M L 4-A L K$ and the resulting aberrant constitutive activity. ${ }^{46}$ There are also other less frequent fusion partners of $A L K$, such as TFG and KIF5B, which mediate ligand-independent dimerization, and therefore constitutive activity of the $A L K$ tyrosine kinase domain. ${ }^{47,48}$ In cell line and mouse models, $E M L 4-A L K$ is highly oncogenic, activates the phosphatidylinositol-3-kinases-Akt and mitogen-activated protein kinase-extracellular signal-regulated kinase pathways, and induces lung tumors. ${ }^{45}$ Clinical patients' characteristics, including never or light smoking history, young age, and adenocarcinoma histology with signet ring cells seem to be associated with NSCLC tumors with a higher probability to detect $A L K$ translocations. ${ }^{49-53}$ In never or light smokers with NSCLC, the prevalence of $A L K$ translocations may be as high as $20 \%-30 \% .{ }^{52} A L K$ translocations are usually mutually exclusive with $E G F R$ or $K R A S$ mutations and predict for a poor response to EGFRTKIs, less responsiveness to platinum-based chemotherapy, and a lower OS in patients with advanced NSCLC. ${ }^{52,54}$ The evidence of EML4-ALK in lung tumors has been detected by FISH, which appears to be the most clinically applicable test. ${ }^{47,51,52,54}$

Crizotinib (PF0234-1066) is an oral adenosine triphosphatecompetitive selective inhibitor of the $A L K$ and MET tyrosine kinases that inhibits tyrosine phosphorylation of activated $A L K{ }^{55}$ In a Phase I-II study, the escalated doses of crizotinib were from $50 \mathrm{mg}$ once daily to $300 \mathrm{mg}$ twice daily, using a standard dose-escalation design. Dose-limiting fatigue in the cohort receiving $300 \mathrm{mg}$ twice daily led to the establishment of a regimen of $250 \mathrm{mg}$ twice daily as the maximum tolerated dose. The expanded cohort with FISH-positive results for $A L K$ rearrangement received $250 \mathrm{mg}$ twice daily as long as they did not have progressive disease or intolerable side effects. A total of 82 patients with advanced $A L K$-positive NSCLC received crizotinib with the mean duration of treatment of 6.4 months and an ORR of $57 \%$, while in $33 \%$ of patients a stable disease was detected. The estimated probability of 6-month PFS was $72 \%$, with no median PFS for the study reached. In terms of safety, grade 1 nausea and diarrhea were the adverse events most commonly reported, and grade 3 or 
Table I Frequency of main genetic abnormalities in non-small cell lung cancer $62-65$

\begin{tabular}{llll}
\hline Gene & Event type & Adenocarcinoma (\%) & Squamous cell carcinoma (\%) \\
\hline EGFR & Mutation & $5-15$ & $<5$ \\
EML4-ALK & Fusion & $5-15$ & 1 \\
KRAS & Mutation & $15-38$ & $<5$ \\
FGFRI & Amplification & 1 & $20-25$ \\
FGFR2 & Mutation & 18 & 5 \\
PIK3CA & Mutation & $2-8$ & 9 \\
PTEN & Mutation/deletion & 2 & 18 \\
CDKN2A & Deletion/mutation & 15 & 45 \\
PDGFRA & Amplification/mutation & $4-9$ & 9 \\
BRAF & Mutation & $<5$ & 3 \\
DDR2 & Mutation & 1 & 4 \\
\hline
\end{tabular}

4 transaminase elevation was the most commonly detected serious adverse event. To date, median OS was not reached..$^{56}$ Additional data from an expanded cohort of 116 patients with a median follow-up of 11 months were reported. ORR was $61 \%$, including two complete and 69 partial responses, and clinical benefit rate was $88 \%$. ORRs were reached rapidly with a median of 8 weeks to response. Preliminary median response duration estimate was 48 weeks. Preliminary median PFS was 10 months. The benefit was consistent across the line of therapy, sex, age, and general fitness. ${ }^{57}$ These results led to ongoing trials which are investigating crizotinib in first-line and second-line advanced $A L K$-positive NSCLC patients. Preliminary data of a large Phase II study which recently closed its accrual have been presented. The data are related to the first 136 enrolled patients who were evaluable for safety and 76 for activity. The majority of them had received at least two prior systemic therapy regimens (93\%). At the time of this analysis, patients had received a median of 9 weeks of crizotinib treatment and $88 \%$ remained on therapy. A waterfall plot of tumor measurements in evaluable patients showed target lesion shrinkage in approximately $90 \%$ of patients (41 patients had $\geq 30 \%$ shrinkage). ${ }^{58}$

The final results of the ongoing trials investigating crizotinib in patients with advanced NSCLC with $A L K$ rearrangements give the real impact in the clinical practice of this biomarker and its inhibitor.

\section{Conclusion}

To date, two biomarkers are available for the personalized medicine in the treatment of advanced NSCLC patients. The EGFR activating mutation has already entered the clinical practice, while the $A L K$ gene fusion is about to. This implies that, whenever possible, an adequate tumor sample tissue for molecular characterization has to be obtained at the moment of the initial diagnosis to start treatment off with the most appropriate therapeutic strategy. The clinical characteristics strongly related with the presence of these biomarkers should guide the selection of patients who must be investigated for their detection. An important issue is that evidence shows that ORR in $E G F R$-mutated or $A L K$-positive patients was not $100 \%$ and, unfortunately, patients who initially benefited from the specific inhibitors experienced - at a certain point of their illness - a progression of their disease. Possible explanations could be the presence of a primary resistance due to the contemporary activation of other pathways which bypass the EGFR pathway, or of an acquired resistance due to the occurrence, during EGFR-TKI therapy, of an additional EGFR gene mutation. The most studied mutation occurs in exon 20 , with the substitution of a threonine for methionine at position 790 (T790M), which is supposed to change the conformation of the receptor and block the binding of gefinitib or erlotinib to the active site, creating resistance to these EGFR-TKIs. ${ }^{59}$ In contrast to the reversible TKIs like gefitinib and erlotinib, the second generation EGFR inhibitors, the irreversible TKIs, among which afatinib (BIBW2992) is in advanced phase of clinical development in NSCLC patients, seem to effectively inhibit EGFR T790M and block the growth of NSCLC cell lines harboring T790M mutations. ${ }^{60}$

Preliminary studies revealed that also in the case of $A L K$ rearrangements, the onset of secondary acquired mutations in the kinase domain of EML4-ALK confer resistance to the specific inhibitor crizotinib. ${ }^{61}$ The interesting retrospective results from the FLEX trial seem to open a new therapeutic option for patients with $E G F R$ IHC-positive tumors (score $\geq 200$ ). If these data are further confirmed, a new therapeutic personalized approach will be available in the clinical practice. Further biomarkers are under investigation for the targeted therapy of both adenocarcinoma and squamous cell carcinoma of the lung, with several trials ongoing and relative results still pending (Table 1). ${ }^{62-65}$ 
To date, considering the EGFR activating mutations and the $E M L 4-A L K$ gene fusion, which is generally mutually exclusive, and the availability of the correspondent EGFRTKIs and crizotinib, about $20 \%$ to $50 \%$ of advanced NSCLC could now be treated in Western and Eastern countries, respectively, with a really targeted therapy.

\section{Disclosure}

Dr Antonio Rossi and Dr Domenico Galetta received honoraria from AstraZeneca and Roche as members of the speakers' bureau.

\section{References}

1. Herbst RS, Heymach JV, Lippman SM. Lung cancer. $N$ Engl J Med. 2008;359(13):1367-1380.

2. Govindan R, Page N, Morgensztern D, et al. Changing epidemiology of small-cell lung cancer in the United States over the last 30 years: analysis of the surveillance, epidemiologic, and end results database. J Clin Oncol. 2006;24(28):4539-4544.

3. Perez-Soler R. HER1/EGFR targeting: refining the strategy. Oncologist. 2004;9(1):58-67.

4. Rossi A, Bria E, Maione P, Palazzolo G, Falanga M, Gridelli C. The role of cetuximab and other epidermal growth factor receptor monoclonal antibodies in the treatment of advanced non-small cell lung cancer. Rev Recent Clin Trials. 2008;3(3):217-227.

5. Lynch TJ, Bell DW, Sordella R, et al. Activating mutations in the epidermal growth factor receptor underlying responsiveness of non-small-cell lung cancer to gefitinib. N Engl J Med. 2004;350(21):2129-2139.

6. Paez JG, Janne PA, Lee JC, et al. EGFR mutations in lung cancer: correlation with clinical response to gefitinib therapy. Science. 2004; 304(5676): 1497-1500.

7. Pao W, Miller V, Zakowski M, et al. EGF receptor gene mutations are common in lung cancers from "never smokers" and are associated with sensitivity of tumors to gefitinib and erlotinib. Proc Natl Acad Sci USA. 2004;101(36):13306-13311.

8. Sequist LV, Bell DW, Lynch TJ, Haber DA. Molecular predictors of response to epidermal growth factor receptor antagonists in non-smallcell lung cancer. J Clin Oncol. 2007;25(5):587-595.

9. Shigematsu H, Lin L, Takahashi T, et al. Clinical and biological features associated with epidermal growth factor receptor gene mutations in lung cancers. J Natl Cancer Inst. 2005;97(5):339-346.

10. Tokumo M, Toyooka S, Kiura K, et al. The relationship between epidermal growth factor receptor mutations and clinicopathologic features in non-small cell lung cancers. Clin Cancer Res. 2005;11(3): $1167-1173$.

11. Nguyen KS, Kobayashi S, Costa DB. Acquired resistance to epidermal growth factor receptor tyrosine kinase inhibitors in non-small-cell lung cancers dependent on the epidermal growth factor receptor pathway. Clin Lung Cancer. 2009;10(4):281-289.

12. Sharma SV, Bell DW, Settleman J, Haber DA. Epidermal growth factor receptor mutations in lung cancer. Nat Rev Cancer. 2007;7(3): 169-181.

13. Yun CH, Boggon TJ, Li Y, et al. Structures of lung cancer-derived EGFR mutants and inhibitor complexes: mechanism of activation and insights into differential inhibitor sensitivity. Cancer Cell. 2007;11(3): $217-227$.

14. Giaccone G, Herbst RS, Manegold C, et al. Gefitinib in combination with gemcitabine and cisplatin in advanced non-small-cell lung cancer: a phase III trial - INTACT 1. J Clin Oncol. 2004;22(5):777-784.

15. Herbst RS, Giaccone G, Schiller JH, et al. Gefitinib in combination with paclitaxel and carboplatin in advanced non-small-cell lung cancer: a phase III trial - INTACT 2. J Clin Oncol. 2004;22(5):785-794.
16. Herbst RS, Prager D, Hermann R, et al. TRIBUTE: a phase III trial of erlotinib hydrochloride (OSI-774) combined with carboplatin and paclitaxel chemotherapy in advanced non-small-cell lung cancer. J Clin Oncol. 2005;23(25):5892-5899.

17. Gatzemeier U, Pluzanska A, Szczesna A, et al. Phase III study of erlotinib in combination with cisplatin and gemcitabine in advanced non-small-cell lung cancer: the Tarceva Lung Cancer Investigation Trial. J Clin Oncol. 2007;25(12):1545-1552.

18. Thatcher N, Chang A, Parikh P, et al. Gefitinib plus best supportive care in previously treated patients with refractory advanced nonsmall-cell lung cancer: results from a randomised, placebo-controlled, multicentre study (Iressa Survival Evaluation in Lung Cancer). Lancet. 2005;366(9496):1527-1537.

19. Shepherd FA, Rodrigues Pereira J, Ciuleanu T, et al. Erlotinib in previously treated non-small-cell lung cancer. $N$ Engl J Med. 2005;353(2): $123-132$.

20. Kim ES, Hirsch V, Mok T, et al. Gefitinib versus docetaxel in previously treated non-small-cell lung cancer (INTEREST): a randomised phase III trial. Lancet. 2008;372(9652):1809-1918.

21. Costa DB, Kobayashi S, Tenen DG, Huberman MS. Pooled analysis of the prospective trials of gefitinib monotherapy for EGFR-mutant non-small cell lung cancers. Lung Cancer. 2007;58(1):95-103.

22. MokTS, Wu YL, Thongprasert S, et al. Gefitinib or carboplatin-paclitaxel in pulmonary adenocarcinoma. $N$ Engl J Med. 2009;361(10):947-957.

23. Fukuoka M, Wu YL, Thongprasert S, et al. Biomarker analyses and final overall survival results from a phase III, randomized, open-label, first-line study of gefitinib versus carboplatin/paclitaxel in clinically selected patients with advanced non-small-cell lung cancer in Asia (IPASS). J Clin Oncol. 2011;29(21):2866-2874.

24. Han JY, Park K, Kim SW, et al. First-SIGNAL: First-line single-agent iressa versus gemcitabine and cisplatin trial in never-smokers with adenocarcinoma of the lung. $J$ Clin Oncol. 2012;30(10):1122-1128.

25. Mitsudomi T, Morita S, Yatabe Y, et al. Gefitinib versus cisplatin plus docetaxel in patients with non-small-cell lung cancer harbouring mutations of the epidermal growth factor receptor (WJTOG3405): an open label, randomised phase 3 trial. Lancet Oncol. 2010;11(2): $121-128$.

26. Maemondo M, Inoue A, Kobayashi K, et al. Gefitinib or chemotherapy for non-small-cell lung cancer with mutated EGFR. NEngl J Med. 2010; 362(25):2380-2388.

27. Inoue $\mathrm{A}$, Kobayashi $\mathrm{K}$, Maemondo $\mathrm{M}$, et al. Final overall survival results of NEJ002, a phase III trial comparing gefitinib to carboplatin (CBDCA) plus paclitaxel (TXL) as the first-line treatment for advanced non-small cell lung cancer (NSCLC) with EGFR mutations [abstract]. J Clin Oncol. 2011;29(Suppl 15):480s.

28. Zhou C, Wu TL, Chen G, et al. Erlotinib versus chemotherapy as first-line therapy for patients with advanced EGFR mutation-positive non-small-cell lung cancer (OPTIMAL, CTONG-0802): a multicentre, open-label, randomised, phase III study. Lancet Oncol. 2011;12(8): 735-742.

29. Rosell R, Carcereny E, Gervais R, et al. Erlotinib versus standard chemotherapy as first-line treatment for European patients with advanced EGFR mutation-positive non-small-cell lung cancer (EURTAC): a multicentre, open-label, randomised phase 3 trial. Lancet Oncol. 2012;13(3):239-246.

30. Lynch TJ, Patel T, Dreisbach L, et al. Cetuximab and first-line taxane/ carboplatin chemotherapy in advanced non-small-cell lung cancer: results of the randomized multicenter phase III trial BMS099. J Clin Oncol. 2010;28(6):911-917.

31. Khambata-Ford S, Harbison CT, Hart LL, et al. Analysis of potential predictive markers of cetuximab benefit in BMS099, a phase III study of cetuximab and first-line taxane/carboplatin in advanced non-smallcell lung cancer. J Clin Oncol. 2010;28(6):918-927.

32. Pirker R, Pereira JR, Szczesna A, et al. Cetuximab plus chemotherapy in patients with advanced non-small-cell lung cancer (FLEX): an open-label randomised phase III trial. Lancet. 2009;373(9674): $1525-1531$. 
33. O’Byrne KJ, Gatzemeier U, Bondarenko I, et al. Molecular biomarkers in non-small-cell lung cancer: a retrospective analysis of data from the phase 3 FLEX study. Lancet Oncol. 2011;12(8):795-805.

34. Cappuzzo F, Hirsch FR, Rossi E, et al. Epidermal growth factor receptor gene and protein and gefitinib sensitivity in non-small-cell lung cancer. J Natl Cancer Inst. 2005;97(9):643-655.

35. Hirsch FR, Varrella-Garcia M, Dziadziuszko R, et al. Fluorescence in situ hybridization subgroup analysis of TRIBUTE, a phase III trial of erlotinib plus carboplatin and paclitaxel in non-small cell lung cancer Clin Cancer Res. 2008;14(19):6317-6323.

36. Zhu CQ, da Cunha Santos C, Ding K, et al. Role of KRAS and EGFR as biomarkers of response to erlotinib in National Cancer Institute of Canada Clinical Trials Group Study BR.21. J Clin Oncol. 2008;26(26): 4268-4275.

37. Hirsch FR, Varella-Gracia M, Bunn PA Jr, et al. Molecular predictors of outcome with gefitinib in a phase III placebo-controlled study in advanced non-small-cell lung cancer. J Clin Oncol. 2006;24(31): 5034-5042.

38. Douillard JY, Shepherd FA, Hirsh V, et al. Molecular predictors of outcome with gefitinib and docetaxel in previously treated non-smallcell lung cancer: data from the randomized phase III INTEREST trial. J Clin Oncol. 2010;28(5):744-752.

39. Cappuzzo F, Ligorio C, Janne PA, et al. Prospective study of gefitinib in epidermal growth factor receptor fluorescence in situ hybridizationpositive/phospho-Akt-positive or never smoker patients with advanced non-small-cell lung cancer: the ONCOBELL trial. J Clin Oncol. 2007; 25(16):2248-2255.

40. Crino L, Cappuzzo F, Zatloukal P, et al. Gefitinib versus vinorelbine in chemotherapy-naive elderly patients with advanced non-small-cell lung cancer (INVITE): a randomized, phase II study. J Clin Oncol. 2008;26(26):4253-4260.

41. Dahabreh IJ, Linardou H, Kosmidis P, Bafaloukos D, Murray S. EGFR gene copy number as a predictive biomarker for patients receiving tyrosine kinase inhibitor treatment: a systematic review and meta-analysis in non-small-cell lung cancer. Ann Oncol. 2011;22(3):545-552.

42. Varrella-Garcia M, Diebold J, Eberhard DA, et al. EGFR fluorescence in situ hybridisation assay: guidelines for application to non-small-cell lung cancer. J Clin Pathol. 2009;62(11):970-977.

43. Tsao MS, Sakurada A, Cutz JC, et al. Erlotinib in lung cancer-molecular and clinical predictors of outcome. N Eng J Med. 2005;353(2):133-144.

44. Pirker R, Pereira JR, von Pawel J, et al. EGFR expression as a predictor of survival for first-line chemotherapy plus cetuximab in patients with advanced non-small-cell lung cancer: analysis of data from the phase 3 FLEX study. Lancet Oncol. 2012;13(1):33-42.

45. Soda M, Choi YL, Enomoto M, et al. Identification of the transforming EML4-ALK fusion gene in non-small-cell lung cancer. Nature. 2007; 448(7153):561-566.

46. Sasaki T, Rodig SJ, Cirieac LR, Janne PA. The biology and treatment of EML4-ALK non-small cell lung cancer. Eur J Cancer. 2010;46(10): 1773-1780.

47. Takeuchi K, Choi YL, Togashi Y, et al. KIF5B-ALK, a novel fusion oncokinase identified by an immunohistochemistry-based diagnostic system for ALK-positive lung cancer. Clin Cancer Res. 2009;15(9): 3143-3149.

48. Rikova K, Guo A, Zeng Q, et al. Global survey of phosphotyrosine signaling identifies oncogenic kinases in lung cancer. Cell. 2007;131(6): 1190-1203.

Current Biomarker Findings

\section{Publish your work in this journal}

Current Biomarker Findings is an international, peer-reviewed, open access journal publishing original research, reports, reviews and commentaries on all areas of biomarker research. The manuscript management system is completely online and includes a very quick and fair
49. Inamura $\mathrm{K}$, Takeuchi $\mathrm{K}$, Togashi $\mathrm{Y}$, et al. EML4-ALK lung cancers are characterized by rare other mutations, a TTF-1 cell lineage, an acinar histology, and young onset. Mod Pathol. 2009;22(4):508-515.

50. Inamura K, Takeuchi K, Togashi Y, et al. EML4-ALK fusion is linked to histological characteristics in a subset of lung cancers. J Thorac Oncol. 2008;3(1):13-17.

51. Rodig SJ, Mino-Kenudson M, Dacic S, et al. Unique clinicopathologic features characterize ALK-rearranged lung adenocarcinoma in the western population. Clin Cancer Res. 2009;15(16):5216-5223.

52. Shaw AT, Yeap BY, Mino-Kenudson M, et al. Clinical features and outcome of patients with non-small-cell lung cancer who harbor EML4-ALK. J Clin Oncol. 2009;27(26):4247-4253.

53. Koivunen JP, Mermel C, Zejnullahu K, et al. EML4-ALK fusion gene and efficacy of an ALK kinase inhibitor in lung cancer. Clin Cancer Res. 2008;14(13):4275-4283.

54. Wong DW, Leung EL, So KK, et al. The EML4-ALK fusion gene is involved in various histologic types of lung cancers from nonsmokers with wild-type EGFR and KRAS. Cancer. 2009;115(8):1723-1733.

55. Christensen JG, Zou HY, Arango ME, et al. Cytoreductive antitumor activity of PF-2341066, a novel inhibitor of anaplastic lymphoma kinase and c-Met, in experimental models of anaplastic large-cell lymphoma. Mol Cancer Ther. 2007;6(12 Pt 1):3314-3322.

56. Kwak EL, Bang YJ, Camidge DR, et al. Anaplastic lymphoma kinase inhibition in non-small-cell lung cancer. N Engl J Med. 2010;363(18): 1693-1703.

57. Camidge DR, Bang Y, Kwak EL, et al. Progression-free survival (PFS) from a phase I study of crizotinib (PF-02341066) in patients with ALKpositive non-small cell lung cancer (NSCLC) [abstract]. J Clin Oncol. 2011;29(Suppl 15):165s.

58. Riely GJ, Kim DW, Crino L, et al. Phase II data for crizotinib (PF 02341066) in ALK-positive advanced non-small cell lung cancer (NSCLC): profile 1005 [abstract]. J Thorac Oncol. 2011; 6(Suppl 6):S411.

59. Pao W, Miller WA, Politi KA, et al. Acquired resistance of lung adenocarcinomas to gefitinib or erlotinib is associated with a second mutation in the EGFR kinase domain. PLoS Med. 2005;2(3):e73.

60. Spicer JF, Rudman SM. EGFR inhibitors in non-small cell lung cancer (NSCLC): the emerging role of the dual irreversible EGFR/ HER2 inhibitor BIBW 2992. Target Oncol. 2010;5(4):245-255.

61. Choi YL, Soda M, Yamashita Y, et al. EML4-ALK mutations in lung cancer that confer resistance to ALK inhibitors. N Engl J Med. 2010; 363(18):1734-1739

62. Ding L, Getz G, Wheeler DA, et al. Somatic mutation affect key pathways in lung adenocarcinoma. Nature. 2008;455(7216):1069-1075.

63. Pao W, Girard N. New driver mutations in non-small-cell lung cancer. Lancet Oncol. 2011;12(2):175-180.

64. Kris MG, Arcila ME, Lau C, et al. Two year results of LCMAP: an institutional program to routinely profile tumor specimens for the presence of mutations in targetable pathways in all patients with nonsmall cell lung cancers [abstract]. J Thorac Oncol. 2011;6(Suppl 2): S346-S347.

65. Hammerman P, Sivachenko A, Pho N, et al. Genomic characterization and targeted therapeutics in squamous cell carcinoma [abstract]. J Thorac Oncol. 2011;6(Suppl 2):S39.

peer-review system. Visit http://www.dovepress.com/testimonials.php to read real quotes from published authors. 